\title{
BMJ Open Can a pathological model improve the abilities of the paretic hand in hemiplegic children? The PAM-AOT study protocol of a randomised controlled trial
}

\author{
Jessica Verzelloni, ${ }^{1}$ Antonino Errante, ${ }^{2}$ Laura Beccani, ${ }^{3}$ Mariacristina Filippi, ${ }^{3}$ \\ Barbara Bressi (D) , ${ }^{4,5}$ Silvio Cavuto, ${ }^{6}$ Settimio Ziccarelli, ${ }^{2}$ Francesca Bozzetti, ${ }^{7}$ \\ Stefania Costi (D) , ${ }^{1,8}$ Elena Pineschi, ${ }^{3}$ Leonardo Fogassi, ${ }^{2}$ Adriano Ferrari ${ }^{1,3}$
}

To cite: Verzelloni J, Errante A, Beccani L, et al. Can a pathological model improve the abilities of the paretic hand in hemiplegic children? The PAM-AOT study protocol of a randomised controlled trial. BMJ Open 2021;11:e053910. doi:10.1136/ bmjopen-2021-053910

- Prepublication history and additional supplemental material for this paper are available online. To view these files, please visit the journal online (http://dx.doi.org/10.1136/ bmjopen-2021-053910).

Received 03 June 2021 Accepted 01 December 2021

Check for updates

(C) Author(s) (or their employer(s)) 2021. Re-use permitted under CC BY-NC. No commercial re-use. See rights and permissions. Published by BMJ.

For numbered affiliations see end of article.

Correspondence to Professor Adriano Ferrari; adriano.ferrari@unimore.it

\section{ABSTRACT}

Introduction Action Observation Treatment (AOT) is an innovative therapeutic approach consisting in the observation of actions followed by subsequent repetition. In children with unilateral cerebral palsy (UCP), it improves upper limb function in daily activities. The standard paradigm of AOT requires the observation of healthy models; however, it has been demonstrated that the mirror neuron system of children with UCP is more activated by observation of pathological models, showing a similar motor repertoire, than by the healthy model, suggesting that AOT based on pathological models is superior to the standard paradigm of $\mathrm{AOT}$ in the functional rehabilitation of the affected upper limb of children with UCP.

Methods and analysis This protocol describes an active two-arm randomised controlled evaluator-blinded trial. Twenty-six children with UCP will participate in 3 weeks of intensive $\mathrm{AOT}$ : the experimental group will observe a pathological model, while the control group will observe a typically developed model. The primary outcome is the spontaneous use of the paretic hand, measured with the Assisting Hand Assessment. Secondary outcome measures are the Melbourne Assessment of Unilateral Upper Limb Function, the ABILHAND-Kids and the Activities Scale for Kids-performance. Assessments will be performed at baseline (TO), at the end of intensive AOT (T1), at 8-12 weeks (T2) and at 24-28 weeks (T3) after the end of intensive AOT.

Ethics and dissemination The trial was approved by the Area Vasta Emilia Nord Ethics Committee (AVEN prot. n. 133117, 29 November 2018), and it was prospectively registered on ClinicalTrials.gov. The results will be submitted for publication to a peer-reviewed journal, discussed with parents of children participating in the trial and disseminated at suitable conferences.

Trial registration number NCT04088994; Pre-results.

\section{INTRODUCTION}

In non-human primates, Action Observation is coded by mirror neurons, which activate during observation and execution of
Strengths and limitations of this study

- This study protocol is the first to test the superiority of a pathological ameliorative model compared with a typically developed model in the 'observation to imitate' paradigm to improve motor learning.

- Based on this assumption, an Action Observation Treatment for children with unilateral cerebral palsy was developed according to the motor repertoire of the paretic hand, in order to match the range of children's capabilities.

- Changes in the activation of their mirror neuron system will be verified in a subgroup of participants through functional MRI.

- As with all studies that require active collaboration from enrolled children, adherence to study procedures may not be adequate; this will, therefore, be monitored throughout the study.

- As the study was designed as a highly specialised intervention, its repeatability in other contexts could be limited.

goal-directed actions. ${ }^{1}$ The existence of a mirror neuron system (MNS) has also been demonstrated in humans; this neural network includes the rostral region of the inferior parietal lobule, the inferior sector of the precentral gyrus (ventral premotor cortex) and the caudal part of the inferior frontal gyrus. ${ }^{2}$ Differently from monkeys, where mirror neurons are not activated by intransitive actions, the MNS in humans is also activated by the observation of pantomimes. ${ }^{3}$ It is also well established that the MNS is directly involved in the recognition of the intention underlying the observed action. ${ }^{45}$

The discovery and study of the MNS may have important implications for human motor rehabilitation, among which the 
development of Action Observation Treatment (AOT).$^{67}$ This therapeutic approach consists in the observation of actions and subsequently repeating them. AOT is based on the 'observation to imitate' paradigm, according to which the observed actions induce an automatic activation of the motor system, which would improve motor learning. ${ }^{89}$

AOT training produces greater motor learning than simple repetitive motor training. ${ }^{10}$ This approach has been clinically tested, with positive results obtained in the treatment of the paretic upper limb (UL) in patients suffering from ischaemic stroke, ${ }^{7}$ also showing the formation of motor memory after training. ${ }^{611} 12$ The effectiveness of AOT has also been demonstrated in the rehabilitation of patients with Parkinson's disease, showing an improvement in freezing during gait, a reduction in bradykinesia and an improvement in activities of daily living. ${ }^{13-17}$ Moreover, AOT plus conventional physiotherapy have been proven to be beneficial in the rehabilitation of patients after major musculoskeletal surgery of lower limbs. ${ }^{18}$

Increasing evidence has shown that a mirror mechanism matching action execution and action observation might be present during infancy, and this neural substrate is similar to that found in adults. ${ }^{19}$ The strongest evidence for a neural signature of action observation in children comes from studies exploring the desynchronisation of the mu-rhythm, which was reported for observation and execution of goal-directed reach and grasp. ${ }^{20}{ }^{21}$ These studies suggest that a direct visual-motor matching process is already detectable at as early as 6 months, suggesting the presence of a matching between action observation and execution already in early infancy. Furthermore, a recent functional MRI (fMRI) study ${ }^{22}$ conducted on adults and children while observing complex grasping actions showed that the activation of MNS in children was less lateralised than that of adults, with a linear growth of lateralisation index as a function of age. Altogether, these studies support the use of AOT as a successful tool for rehabilitation in children.

The first study reporting the effectiveness of AOT in the UL motor rehabilitation in paediatric patients was conducted on children with cerebral palsy. ${ }^{23}$ Subsequently, the consistency of the results obtained with AOT in the motor rehabilitation of the UL was demonstrated in a randomised controlled trial conducted on a cohort of children with unilateral cerebral palsy (UCP).$^{24}$ Recently, two systematic reviews regarding the effectiveness of AOT in the UL rehabilitation of children with cerebral palsy were published: the first emphasises that the variability between studies does not lead to any clear conclusion regarding the effectiveness of this approach, ${ }^{25}$ while the second suggests that AOT is a promising intervention for UL rehabilitation in children with UCP. ${ }^{26}$

Standard AOT consists in the observation of a typically developed model (TDM); however, an important assumption of AOT is that the MNS activates when the visual description of the observed action is matched with its corresponding motor representation in the observer's brain (direct matching hypothesis). ${ }^{27}$ In fact, it has been shown that while observed actions belonging to the behavioural repertoire of the observer are mapped on his/her motor system, ${ }^{28}$ those that belong only to her/ his visual experience are only categorised based on visual characteristics, without inducing any motor resonance phenomenon in the observer's brain. ${ }^{29}$ Therefore, given the differences in their motor repertoire, children with UCP might have a reduced activation of the MNS during observation of actions performed by healthy subjects. Based on this hypothesis, a recent fMRI study ${ }^{30}$ demonstrated that, compared with the observation of a TDM, the activation of the MNS in children with UCP was stronger during the observation of goal-directed actions performed by a pathological ameliorative model (PAM). The PAM consists in a paretic UL having a motor repertoire similar to the observer's, but with higher level of performance. ${ }^{30}$ Thus, the aim of the PAM-AOT trial is to verify the superiority of AOT performed with a PAM over AOT performed with a TDM in the functional rehabilitation of UL of children with UCP. To do so, we will evaluate the short-term, medium-term and long-term clinical effects of PAM-AOT compared with TDM-AOT on bimanual hand function, quality of unilateral UL movements, manual ability and performance in activities of daily living.

\section{METHODS AND ANALYSIS \\ Study design}

This is the study protocol of a randomised controlled evaluator-blinded trial with two active arms. After randomisation, the participants will undergo 3 weeks of intensive AOT based on either the PAM (experimental group) or the TDM (control group). Figure 1 represents the flowchart of the study.

Randomisation will be stratified according to the functional classification of the paretic hand of participants, that is, levels $4-5$ and levels $6-7$ of the House Functional Classification System. ${ }^{31}{ }^{32}$ Sequence generation will be performed by random number generator, with a 1:1 allocation ratio. Allocation will be concealed by central randomisation, which will be performed by an independent physiotherapist (StC), who has no direct contact with the clinical aspects of the trial and will assign participants to the study arms.

\section{Participants}

Children and adolescents with UCP treated by the special Unit for Children Rehabilitation of the Santa Maria Nuova Hospital (UDGEE, AUSL-IRCCS di Reggio Emilia, Italy) will be screened for eligibility by rehabilitation professionals of the Unit (JV, LB, BB and AF). Eligible children and adolescents and their parents will be thoroughly informed about the purposes and methods of the study and invited to participate. Before enrolment, informed consent (online supplemental additional file 1) will be requested from parents and from those participants able to grant it (table 1). 


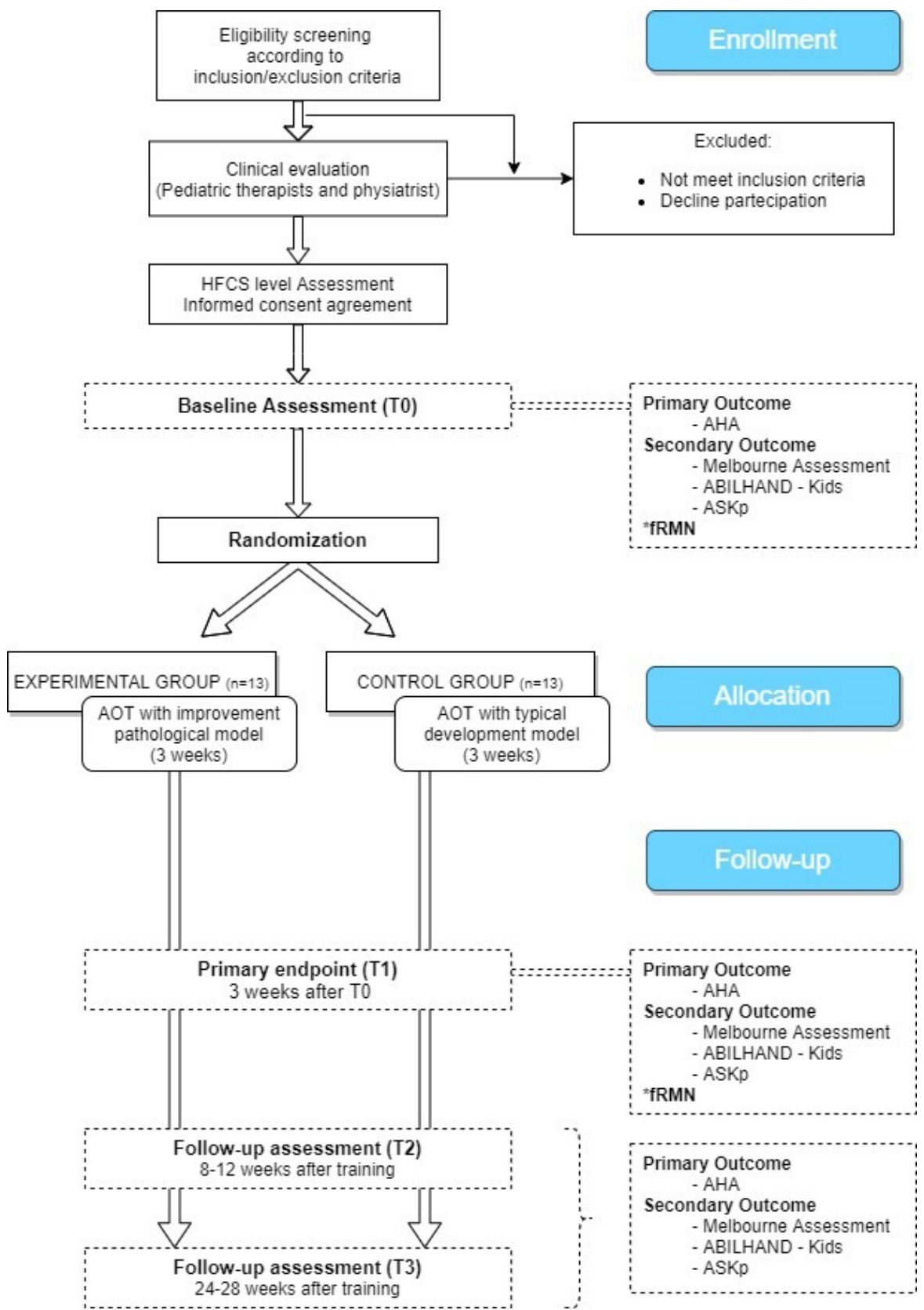

Figure 1 Flowchart of PAM-AOT study according to CONSORT guidelines. AHA, Assisting Hand Assessment; AOT: Action Observation Treatment; ASKp, Activities Scale for Kids, performance version; CONSORT, Consolidated Standards of Reporting Trials; fMRI: functional MRI; PAM, pathological ameliorative model. *Only in a subgroup of children enrolled.

The inclusion criteria will be:

- age 6-16 years at the time of recruitment, with confirmed diagnosis of UCP according to the 2005 definition of cerebral palsy (supported by MRI and clinical history). ${ }^{33}$

- Predominant spasticity rather than dystonia or weakness interfering with UL function according to the definition of motor type by Sanger et al. ${ }^{34}$

- Mild or moderately severe functional impairment of paretic hand, from level 4 (poor active assist) to level 7 (partial spontaneous use), according to the House Functional Classification System. ${ }^{31} 32$

- Sufficiently cooperative to participate in AOT.

Exclusion criteria will be:
- sensory impairment and/or uncorrected visual impairment of central origin.

- Severe cognitive disability, controlled by Raven's Progressive Colored Matrices. ${ }^{35}$

- Drug-resistant epilepsy.

- Previous orthopaedic surgery on the UL within 8 months prior to study recruitment.

- BoNT-A injection at the UL within 6 months prior to study recruitment.

\section{Sample size}

According to Consolidated Standards of Reporting Trials guidelines, ${ }^{36}{ }^{37}$ the estimated sample size is based on projected treatment effects on the primary outcome 
Table 1 Timeline of study procedures

\begin{tabular}{|c|c|c|c|c|c|}
\hline \multirow[b]{2}{*}{ Timepoint } & \multirow[b]{2}{*}{ T1 } & \multicolumn{4}{|l|}{ Study period } \\
\hline & & T0 (baseline) & $\begin{array}{l}\text { T1 } \\
\text { (3 weeks after T0) }\end{array}$ & $\begin{array}{l}\text { T2 } \\
\text { (8-12 weeks after T1) }\end{array}$ & $\begin{array}{l}\text { T3 } \\
\text { (24-28 weeks after T1) }\end{array}$ \\
\hline \multicolumn{6}{|l|}{ Enrolment } \\
\hline Eligibility screening & $x$ & & & & \\
\hline Informed consent & $x$ & & & & \\
\hline AOT treatment & & $\longleftarrow$ & $\longrightarrow$ & & \\
\hline \multicolumn{6}{|l|}{ Assessments } \\
\hline HFCS & $x$ & & & & \\
\hline ASKp & & $x$ & $x$ & $x$ & $x$ \\
\hline fMRI & & $x$ & $x$ & & \\
\hline
\end{tabular}

AHA, Assisting Hand Assessment; AOT, action observation treatment; ASKp, Activities Scale for Kids, performance version; fMRI, functional MRI; HFCS, house functional classification system; MUUL, Melbourne Assessment of Unilateral Upper Limb Function.

measure, the Assistive Hand Assessment (AHA) score. The responsiveness of this scale to change has been estimated by Eliasson et al, who reported an effect size of 1.16 in a population of children with UCP. ${ }^{38}$ Preliminary statistical analysis (Software nQueryAdvisor) indicates that, to detect an effect size of 1.16, with a significance level of $0.05 \%$ and $80 \%$ of statistical power, a minimum sample size of 13 participants per group is required.

\section{Outcomes and measurements}

The primary outcome measure to assess the superiority of the pathological model over the TDM is the change in spontaneous use of the paretic hand from baseline to follow-up. This outcome will be measured using the AHA (V.4.4), ${ }^{39}$ which is a performance-based test developed for children with hemiplegic cerebral palsy. The AHA is administered through a semistructured play session, which requires bimanual handling and lasts $15 \mathrm{~min}$. The smallest detectable difference over time has indicated that a change in AHA scores must be 3.65 (0.76 logits) or more to be considered a significant change with $95 \%$ probability. ${ }^{39} 40$

Secondary outcomes are: (a) quality of the unilateral UL movements in children with neurological impairments, measured by the Melbourne Assessment of Unilateral Upper Limb (MUUL) Function. ${ }^{41}$ The MUUL administration lasts about $30 \mathrm{~min}$ and examines the most important components of UL function, such as pointing, reaching, grasping, manipulating, releasing and bringing the hand to the mouth, (b) manual ability, measured by the ABILHAND-Kids, ${ }^{42}$ which is a unidimensional questionnaire focusing on 21 bimanual and unimanual daily activities that are scored according to the child's difficulty as perceived by parents, for children with UL impairment, (c) performance of activities of daily living, measured by the Italian version of the Activities Scale for Kids, performance version (ASKp). ${ }^{43}$ The ASKp is a measure of physical disability designed for children with limitations due to musculoskeletal disorders, which has also been tested in children with cerebral palsy. ${ }^{45}$ The questionnaire focuses on the child's performance of activities that most often happen at home, at school and on the playground. This scale indicates the children's perspectives of their disability and provides the option of examining performance.

Assessments will be blindly performed at baseline (T0), at the end of intensive AOT (T1) and at 8-12 weeks (T2) and 24-28 weeks (T3) after the end of the intensive AOT treatment, as reported in table 1.

\section{Blinding}

Participants and their parents will be informed about the study aims and procedures, but they will be blinded to group allocation. The parents will be invited to stay outside the room during the administration of the intervention; if the child requests the parents be present, they will be seated far from the monitor where the actions will be shown. The physiotherapists performing the intervention will not be blinded to group allocation. Outcome assessments will be administered and scored by a certified physiotherapist (MF) blinded to group allocation. 
Table 2 Examples of the exercise proposed

\begin{tabular}{|c|c|c|c|}
\hline Unimanual & Session A & Session B & Session C \\
\hline $\begin{array}{l}\text { Level } \\
6-7 \text { of HFCS }\end{array}$ & $\begin{array}{l}\text { Remove the lid of a bottle of } \\
\text { paint ('easy-cap' lid). }\end{array}$ & $\begin{array}{l}\text { Grasp the bottle, turn it } \\
\text { upside down, and squeeze } \\
\text { the paint into the dish. }\end{array}$ & $\begin{array}{l}\text { Place hand in the paint, then } \\
\text { leave handprint on the sheet } \\
\text { of paper (adducted fingers). }\end{array}$ \\
\hline $\begin{array}{l}\text { Level } \\
4-5 \text { of HFCS }\end{array}$ & $\begin{array}{l}\text { Remove the cap of a jar of } \\
\text { paint (cylindrical cap). }\end{array}$ & $\begin{array}{l}\text { Grab the jar and squeeze the } \\
\text { paint upside down on the } \\
\text { plate. }\end{array}$ & $\begin{array}{l}\text { Place the hand on the paint } \\
\text { and leave the hand's imprint } \\
\text { (the sheet of paper on a } \\
\text { support). }\end{array}$ \\
\hline $\begin{array}{l}\text { Level } \\
6-7 \text { of HFCS }\end{array}$ & $\begin{array}{l}\text { The less affected hand fixes the } \\
\text { loaf of bread, the paretic hand } \\
\text { cuts a slice. }\end{array}$ & $\begin{array}{l}\text { The paretic hand holds } \\
\text { the slice of bread, the less } \\
\text { affected hand spreads the } \\
\text { chocolate spread. }\end{array}$ & $\begin{array}{l}\text { The less affected hand pours } \\
\text { some sprinkles on the paretic } \\
\text { hand. The paretic hand } \\
\text { sprinkles the sprinkles onto } \\
\text { the slice of bread. }\end{array}$ \\
\hline
\end{tabular}

HFCS, House Functional Classification System.

\section{Active interventions}

The experimental group will be exposed to intensive AOT based on PAM, which consists in watching video sequences of goal-directed actions, and then reproducing the same actions using exactly the same setting and objects. The video sequences have been recorded using a pathological model.

The control group will be exposed to intensive AOT based on the TDM, which consists in watching video sequences of goal-directed actions, and then reproducing the same actions using exactly the same setting and objects. The video sequences have been recorded using a TDM.

Therefore, both groups will be exposed to active interventions, the only difference being the observed model in the video sequences.

Both the active interventions will be carried out 3 days per week for a total duration of 3 weeks, and both will consist in 15 exercises based on activities of daily living of increasing complexity performed with one or both of the ULs. Each exercise consists in three sequential goaldirected actions of increasing complexity. Examples of the exercises proposed are described in table 2. Participants will watch each action for a maximum of 3 min (or as long as attention is held) and will then be requested to perform the observed action exactly at least three times, for a maximum of $3 \mathrm{~min}$, using the same objects and setting shown in the video. Each exercise will take 20-25 min maximum to complete, including the time necessary to change the setting of the different actions. The first eight exercises are unimanual, so as to focus the child's attention on the paretic hand. The remaining seven exercises are bimanual, and the paretic hand can be used as a support of the lessaffected hand or to perform an action symmetrical to that of the less affected hand (eg, using a rolling pin).

In order to match the range of children's capabilities with the activities proposed, two sets of AOT exercises will be created for both models (TDM and PAM) according to the two levels of stratification. Thus, regardless of group allocation, the children in both groups classified as levels 4-5 of paretic hand function will perform a set of exercises that is different from the set for those classified as levels 6-7 (table 2). To guarantee the feasibility of the activity proposed, the exercises in the two sets differ only in terms of the movement requested (ie, type of grasp).

To maximise adherence, the AOT exercises will be customised in terms of activity and/or objects used, based on the participants' sex and age group (6-10 vs 11-16 years).

\section{Setting}

Each action will be video recorded from a first-person perspective, from a shoulder camera angle. Only the setting and the ULs will be included in the frame. This choice avoids confounding elements, allows participants to immediately understand the actions they must repeat and is based on the evidence that MNS is more activated by observation of actions performed in subjective view. ${ }^{4647}$ A specular reflection is carried out for each video to provide all participants with a version of the actions performed by the hand homologous to their paretic one. Thus, children with right hemiplegia will watch a right hand performing unimanual exercises and the main task in bimanual exercises, and vice versa for children with left hemiplegia.

The children will be sitting on a chair, with their arms placed on a table. The seat will be adjusted to have the 
table at waist height. The distance between the shoulders and the table should be as long as the humerus. A 22-inch monitor will be placed in front of the child at a distance of $1 \mathrm{~m}$. Two physiotherapists will prepare the setting and prevent participants from observing the setting arrangement by using an occluding panel. This masking avoids the inadvertent activation of the child's MNS induced by observing the physiotherapist's hand movements. If needed, the physiotherapists can hold the child's attention during task execution using standardised phrases.

\section{Statistical analyses}

The primary endpoint will be analysed using a generalised estimating equations model characterised by the following:

- between-subject factors: randomisation of arm, age and level of functional classification.

- Within-subjects factor: time points (T0, T1, T2 and T3).

- Covariance for the within-subjects factor: in the absence of useful indications a priori, it has been planned to analyse data assuming an 'unstructured' covariance matrix. In case of computational problems, this structure will be replaced by a simpler one (roughly, autoregressive AR).

Each estimate will be accompanied by its bilateral 95\% CI, assuming a normal distribution for the relative estimator. To facilitate interpretation of the comparisons, the effect sizes will be represented in terms of Cohen's D. ${ }^{48}$ The data will be analysed using R or SAS software, based on availability at the time of analysis.

\section{Brain imaging study fMRI experimental design and stimuli}

Before recruitment, participants will be informed about the experimental protocol phases, including also two fMRI sessions, one before and one immediately after the end of the intervention. Volunteer participants suitable to also participate in the fMRI study will be enrolled according to the following additional criteria: (a) sufficiently collaborative to participate in neuroimaging studies lasting approximately $45 \mathrm{~min}$, (b) absence of specific contraindications to performing MRI investigations (implants and metal prostheses, shunts, etc.).

After this selection, only a subgroup of study participants $(\mathrm{N}=16)$, evenly assigned to the experimental and to the control group and matched for age, will undergo the fMRI evaluation.

Before starting each functional imaging session, the participants will complete a training phase lasting about 30 min to allow them to familiarise themselves with the MR system and the experimental procedure. To this end, video clips, illustrative brochures and an MRI simulator, reproducing the real sound effects of the MRI system, will be used during the training session. After the training session, participants will undergo the real MRI session (consisting in four short runs lasting about $7 \mathrm{~min}$ each) and will be instructed to perform two functional tasks:

- action observation (functional runs 1-2).
- motor task (runs 3-4).

During the first two functional runs, the participants will be instructed to observe short video clips (lasting 2 s each), showing different actions performed by an actor with the hand ipsilateral to the child's paretic one, from a first-person perspective. The actions will consist in reaching and grasping an object (eg, a sphere, cube, cylinder, toy car, Lego brick, etc) placed in front of the actor. The objects chosen for the interaction will be of different colours in order to increase the variability of the trials. The observation task includes two experimental conditions: (a) observation of the paretic hand, in which the actions will be performed by another patient with UCP (b) observation of the healthy hand, in which the action will be performed by a TDM. Each participant will observe a specific type of paretic hand, corresponding to that participant's own level of impairment. The visual features of each video will be balanced between the experimental conditions to control for the effects of brightness, contrast, sharpness and the amount of visual information. A total of 24 video clips will be prepared (six objects $\times$ two colours $\times$ two conditions). The observation of a static frame corresponding to each video clip will be used as the control condition (control observation). Each trial of experimental and control conditions will be presented 48 times during the experiment, 24 times for each run.

Each of the two observation runs will be acquired using a block paradigm (total $\mathrm{N}$ of blocks=16), during which participants will observe 10 videos belonging to the same experimental or control condition. Each block will be followed by a variable rest period, lasting 10 or $12 \mathrm{~s}$. The duration of each block will be $20 \mathrm{~s}$. During the rest condition, in the absence of experimental stimuli, a fixation point will be presented (a white cross on a black background) on which the participants must keep their gaze. Each observation run will last approximately $7 \mathrm{~min}$.

The stimuli will be presented binocularly by means of LCD googles (VisuaStim-SVGA-Resonance Technology, USA). In addition, a system of headphones with pneumatic mechanism will be used to reduce the noise caused by the MR system during the session $(30 \mathrm{~dB})$.

In the third and fourth functional runs, the participants will be instructed to perform a motor task with their paretic hand, consisting in the simple manipulation of an object. Plastic or wooden objects will be used for the task inside the MR scanner. The motor task will be acquired using a block paradigm (16 s blocks), alternating with rest periods ( $10 \mathrm{~s}$ or $12 \mathrm{~s})$. The rest condition during the motor task will require the participant to simply remain still, with eyes closed and relaxed. As the control condition, the participant will be instructed to perform a simple opening-closing movement with the impaired hand. Each experimental and control block will be presented eight times, and the overall duration of the task will be approximately 4 min.

It is worth noting that a similar fMRI paradigm was recently used to assess MNS activation in children with UCP. $^{30}$ 
Acquisition of fMRI images, processing and statistical analyses

Morphological and functional images will be acquired using a GE Discovery MR750 3.0T closed MRI scanner located at the University of Parma Hospital (Italy), equipped with a 32-channel head coil. All functional images will be acquired by gradient-echo echo-planar imaging sequence. In addition to the functional images, a high spatial resolution 3D morphological volume (BRAVO MIK) will be acquired.

Preprocessing of functional images will be carried out using SPM V.12 software (Wellcome Department of Imaging Neuroscience, University College, London, UK; http://www.fil.ion.ucl.ac.uk/spm), implementing the spatial realignment, slice-timing correction, anatomicalfunctional coregistration, normalisation to the Montreal Neurological Institute (MNI) template and spatial smoothing. The general linear model will be used to compute group-level analysis, calculating the main effects of the experimental and control conditions ( $t$ contrast). To assess the activation of each experimental condition and the contrast between conditions, a region of interest analysis will be performed at the single-subject level in the main MNS areas, extracting the Blood Oxygenation Level Dependent (BOLD) signal associated with each condition. The Student's t test will be used to assess BOLD signal differences between conditions. All statistical parametric maps will be corrected by applying the familywise error rate method for multiple comparisons, with a significance level of $\mathrm{P}$ (corr.) $<0.001$.

\section{DISCUSSION}

This study compares two very similar AOT approaches for the rehabilitation of children with UCP that, however, differ in their principle. ${ }^{49}$ In fact, the applications of AOT in children rehabilitation have usually been inspired by the typical developed model of a healthy child. However, based on our clinical experience with children with UCP, no rehabilitation approaches can result in the complete recovery of the UL function. Frequently, the best possible improvement consists in adapting the performance of children with CP to meet their developmental needs. Nonetheless, performance remains different from that of average healthy children. If, in the current state of medical knowledge, adaptation is the outcome to be sought for UL functional rehabilitation in children with UCP, perhaps rehabilitation professionals should not strive for 'normality', but rather maximise function by proposing ameliorative yet pathological models. This could mean moving from the current paradigm, which pursues motor learning through the observation of TDM, and adopting a different logic that proposes the most functional of the pathological model attainable, thus respecting the differences imposed by the nature, the extent and the stage of CP.

Typical development tends to replicate itself fairly stably if the culture, community and environment remain homogeneous. The development of CP (natural history) changes rapidly due to the nature of the central nervous system lesions and to the progress in therapeutics, including those achieved in rehabilitation. Thus, we should continuously update our knowledge of the changes in CP practice.

If PAM-AOT is shown to be effective, new rehabilitation strategies based on exercises calibrated to the patient's motor repertoire will be available, in the framework of tailored medicine.

In the present study protocol, PAM-AOT is accompanied by fMRI assessment to investigate the possible subsequent functional changes in the MNS. In particular, this investigation may also permit studying the lateralisation of neural activation during the execution of motor tasks and during action observation. Also, the degree and lateralisation of activation may correlate with improvements in hand motor function parameters obtained through the primary and secondary motor outcome measures. Hopefully, this analysis will make it possible to identify the clinical features of those children with CP who would benefit more from AOT, thus improving the evidence of its effectiveness in specific subgroups of patients.

\section{Data availability statement}

The data set will be stored on a password-protected computer and managed by the Information and Technologies Service (Servizio di tecnologie Informatiche e TelematicheSTIT) of the Azienda USL-IRCCS of Reggio Emilia to protect patient privacy and data.

Neuroimaging data acquired at the University of Parma Hospital will be transferred via (Digital Imaging and COmmunications in Medicine) DICOM protocol to a dedicated server of the University of Parma and will be available to conduct the statistical analyses.

At the beginning of the study, each patient will be assigned an alphanumeric identification code, which does not allow to directly identify the patient's name. All data collected during the study, with the exception of demographic information, will be recorded, processed and stored together with this code. Only the data manager and authorised staff members will be able to associate this code with the patient's name.

All the acquired and analysed data are available from the corresponding author on reasonable request.

\section{Patient and public involvement}

No patient was involved in the development of the research question, the study design, the choice of outcomes measures or the conduction of the study. However, the development of the exercise protocols was based on the analysis of satisfaction questionnaires completed by participants in a previous study. ${ }^{24}$ The results of the study will be shared with the participants and their families by means of dedicated meetings. At the end of the 3 weeks of intensive AOT, through a semistructured interview, we will collect data about satisfaction with the programme from participants and their parents, independently. In 
compliance with the study procedures, and in order to facilitate adherence to the study procedures, the intervention period and the follow-up appointments will be made in agreement with each family.

\section{ETHICS AND DISSEMINATION}

The trial was approved by the Area Vasta Emilia Nord Ethics Committee (AVEN prot. n. 133117, Nov 29, 2018) and was prospectively registered on ClinicalTrials.gov. Any changes to the original protocol will be requested from the Ethics Committee through the appropriate procedure and applied only after its approval. This project began recruitment in December 2019, and we expect to complete data collection in January 2022. The results will be submitted for publication to a peer-reviewed journal, shared with parents and participants to the trial, and disseminated at suitable conferences.

\section{Author affiliations}

${ }^{1}$ Department of Surgery, Medicine, Dentistry and Morphological Sciences, University of Modena and Reggio Emilia, Modena, Emilia-Romagna, Italy

${ }^{2}$ Department of Medicine and Surgery, University of Parma, Parma, Emilia-

Romagna, Italy

${ }^{3}$ Unità per le gravi disabilità dell'età evolutiva, Azienda USL_-IRCCS di Reggio

Emilia, Reggio Emilia, Emilia-Romagna, Italy

${ }^{4}$ Physical Medicine and Rehabilitation Unit, Azienda USL—IRCCS di Reggio Emilia, Reggio Emilia, Emilia-Romagna, Italy

${ }^{5} \mathrm{PhD}$ Program in Clinical and Experimental Medicine, Department of Biomedical, Metabolic and Neural Sciences, University of Modena and Reggio Emilia, Modena, Emilia-Romagna, Italy

${ }^{6}$ Research and Statistics Infrastructure, Azienda USL-IRCCS di Reggio Emilia, Reggio Emilia, Emilia-Romagna, Italy

${ }^{7}$ Neuroradiology Unit, University Hospital of Parma, Parma, Emilia-Romagna, Italy

${ }^{8}$ Scientific Directorate, Azienda USL-IRCCS di Reggio Emilia, Reggio Emilia, EmiliaRomagna, Italy

\section{Twitter Barbara Bressi @barbara_bressi}

Contributors AF, LF, SiC, AE, MF, LB and StC designed the research study. JV, $E P, L B$ and MCF designed the experimental and control interventions of AOT exercises. JV, AE, EP and BB created the video sequences of goal-directed actions. $A F, J V, L B$ and BB are recruiting patients. MCF is blindly administering all outcome assessments. JV and LB are administering experimental and control interventions of $\mathrm{AOT}$. StC is managing the concealed allocation. AE, FB and LF are responsible for the brain imaging study. StC took the lead in writing the manuscript, which was drafted by JV and revised by SiC, BB, AF, AE, FB and LF. All authors have read and approved the final manuscript.

Funding This work was supported by Chiesi Farmaceutici S.p.A., Parma (Italy). Competing interests None declared.

Patient consent for publication Not applicable.

Provenance and peer review Not commissioned; externally peer reviewed.

Supplemental material This content has been supplied by the author(s). It has not been vetted by BMJ Publishing Group Limited (BMJ) and may not have been peer-reviewed. Any opinions or recommendations discussed are solely those of the author(s) and are not endorsed by BMJ. BMJ disclaims all liability and responsibility arising from any reliance placed on the content. Where the content includes any translated material, BMJ does not warrant the accuracy and reliability of the translations (including but not limited to local regulations, clinical guidelines, terminology, drug names and drug dosages), and is not responsible for any error and/or omissions arising from translation and adaptation or otherwise.

Open access This is an open access article distributed in accordance with the Creative Commons Attribution Non Commercial (CC BY-NC 4.0) license, which permits others to distribute, remix, adapt, build upon this work non-commercially, and license their derivative works on different terms, provided the original work is properly cited, appropriate credit is given, any changes made indicated, and the use is non-commercial. See: http://creativecommons.org/licenses/by-nc/4.0/.

\section{ORCID iDs}

Barbara Bressi http://orcid.org/0000-0001-8014-2591

Stefania Costi http://orcid.org/0000-0002-4645-0336

\section{REFERENCES}

1 Gallese V, Fadiga L, Fogassi L, et al. Action recognition in the premotor cortex. Brain 1996;119 (Pt 2:593-609.

2 Rizzolatti G, Cattaneo L, Fabbri-Destro M, et al. Cortical mechanisms underlying the organization of goal-directed actions and mirror neuron-based action understanding. Physiol Rev 2014;94:655-706.

3 Caspers S, Zilles K, Laird AR, et al. ALE meta-analysis of action observation and imitation in the human brain. Neuroimage 2010;50:1148-67.

4 lacoboni M, Molnar-Szakacs I, Gallese V, et al. Grasping the intentions of others with one's own mirror neuron system. PLoS Biol 2005;3:e79.

5 Fogassi L, Ferrari PF, Gesierich B, et al. Parietal lobe: from action organization to intention understanding. Science 2005;308:662-7.

6 Ertelt D, Small S, Solodkin A, et al. Action observation has a positive impact on rehabilitation of motor deficits after stroke. Neuroimage 2007;36 Suppl 2:T164-73.

7 Buccino G. Action observation treatment: a novel tool in neurorehabilitation. Philos Trans $R$ Soc Lond B Biol Sci 2014;369:20130185.

8 Buccino G, Vogt S, Ritzl A, et al. Neural circuits underlying imitation learning of hand actions: an event-related fMRI study. Neuron 2004:42:323-34

9 Pomeroy VM, Clark CA, Miller JSG, et al. The potential for utilizing the "mirror neurone system" to enhance recovery of the severely affected upper limb early after stroke: a review and hypothesis. Neurorehabil Neural Repair 2005;19:4-13.

10 Stefan K, Cohen LG, Duque J, et al. Formation of a motor memory by action observation. J Neurosci 2005;25:9339-46.

11 Celnik P, Webster B, Glasser DM, et al. Effects of action observation on physical training after stroke. Stroke 2008;39:1814-20.

12 Franceschini M, Agosti M, Cantagallo A, et al. Mirror neurons: action observation treatment as a tool in stroke rehabilitation. Eur J Phys Rehabil Med 2010;46:517-23.

13 Pelosin E, Avanzino L, Bove M, et al. Action observation improves freezing of gait in patients with Parkinson's disease. Neurorehabil Neural Repair 2010;24:746-52.

14 Pelosin E, Bove M, Ruggeri P, et al. Reduction of bradykinesia of finger movements by a single session of action observation in Parkinson disease. Neurorehabil Neural Repair 2013;27:552-60.

15 Buccino G, Gatti R, Giusti MC, et al. Action observation treatment improves autonomy in daily activities in Parkinson's disease patients: results from a pilot study. Mov Disord 2011;26:1963-4.

16 Agosta F, Gatti R, Sarasso E, et al. Brain plasticity in Parkinson's disease with freezing of gait induced by action observation training. $J$ Neurol 2017;264:88-101.

17 Abbruzzese G, Marchese R, Avanzino L, et al. Rehabilitation for Parkinson's disease: current outlook and future challenges. Parkinsonism Relat Disord 2016;22 Suppl 1:S60-4.

18 Bellelli G, Buccino G, Bernardini B, et al. Action observation treatment improves recovery of postsurgical orthopedic patients: evidence for a top-down effect? Arch Phys Med Rehabil 2010;91:1489-94.

19 Burzi V, Tealdi G, Boyd RN, et al. Action observation in infancy: implications for neuro-rehabilitation. Dev Med Child Neurol 2016;58 Suppl 4:74-7.

20 Lepage J-F, Théoret $\mathrm{H}$. Eeg evidence for the presence of an action observation-execution matching system in children. Eur J Neurosci 2006;23:2505-10.

21 Marshall PJ, Young T, Meltzoff AN. Neural correlates of action observation and execution in 14-month-old infants: an event-related EEG desynchronization study. Dev Sci 2011;14:474-80.

22 Biagi L, Cioni G, Fogassi L, et al. Action observation network in childhood: a comparative fMRI study with adults. Dev Sci 2016;19:1075-86.

23 Buccino G, Arisi D, Gough P, et al. Improving upper limb motor functions through action observation treatment: a pilot study in children with cerebral palsy. Dev Med Child Neurol 2012;54:822-8.

24 Sgandurra G, Ferrari A, Cossu G, et al. Randomized trial of observation and execution of upper extremity actions versus action 
alone in children with unilateral cerebral palsy. Neurorehabil Neural Repair 2013;27:808-15.

25 Abdelhaleem N, Taher S, Mahmoud M, et al. Effect of action observation therapy on motor function in children with cerebral palsy: a systematic review of randomized controlled trials with metaanalysis. Clin Rehabil 2021;35:51-63.

26 Alamer A, Melese H, Adugna B. Effectiveness of action observation training on upper limb motor function in children with hemiplegic cerebral palsy: a systematic review of randomized controlled trials. Pediatric Health Med Ther 2020;11:335-46.

27 Rizzolatti G, Fogassi L, Gallese V. Neurophysiological mechanisms underlying the understanding and imitation of action. Nat Rev Neurosci 2001;2:661-70.

28 Calvo-Merino B, Glaser DE, Grèzes J, et al. Action observation and acquired motor skills: an fMRI study with expert dancers. Cereb Cortex 2005;15:1243-9.

29 Calvo-Merino B, Grèzes J, Glaser DE, et al. Seeing or doing? Influence of visual and motor familiarity in action observation. Curr Biol 2006;16:1905-10.

30 Errante A, Di Cesare G, Pinardi C, et al. Mirror neuron system activation in children with unilateral cerebral palsy during observation of actions performed by a pathological model. Neurorehabil Neural Repair 2019;33:419-31.

31 House JH, Gwathmey FW, Fidler MO. A dynamic approach to the thumb-in palm deformity in cerebral palsy. J Bone Joint Surg Am 1981;63:216-25.

32 Koman LA, Williams RMM, Evans PJ, et al. Quantification of upper extremity function and range of motion in children with cerebra palsy. Dev Med Child Neurol 2008;50:910-7.

33 Bax M, Goldstein M, Rosenbaum P, et al. Proposed definition and classification of cerebral palsy, April 2005. Dev Med Child Neurol 2005;47:571-6.

34 Sanger TD, Delgado MR, Gaebler-Spira D, et al. Classification and definition of disorders causing hypertonia in childhood. Pediatrics 2003;111:e89-97.

35 Pueyo R, Junqué C, Vendrell P, et al. Raven's coloured progressive matrices as a measure of cognitive functioning in cerebral palsy. $J$ Intellect Disabil Res 2008;52:437-45.

36 Weller C, McNeil J. Consort 2010 statement: updated guidelines can improve wound care. J Wound Care 2010;19:347-53.
37 Schulz KF, Altman DG, Moher D, et al. Consort 2010 statement: updated guidelines for reporting parallel group randomized trials. Ann Intern Med 2010;152:726-32.

38 Eliasson A-C, Krumlinde-sundholm L, Shaw K, et al. Effects of constraint-induced movement therapy in young children with hemiplegic cerebral palsy: an adapted model. Dev Med Child Neurol 2005;47:266-75

39 Holmefur M, Krumlinde-Sundholm L, Eliasson A-C. Interrater and intrarater reliability of the assisting hand assessment. Am J Occup Ther 2007;61:79-84.

40 Sgandurra G, Ferrari A, Cossu G, et al. Upper limb children actionobservation training (UP-CAT): a randomised controlled trial in hemiplegic cerebral palsy. BMC Neurol 2011;11:80.

41 Randall M, Imms C, Carey LM, et al. Rasch analysis of the Melbourne assessment of unilateral upper limb function. Dev Med Child Neurol 2014;56:665-72.

42 Arnould C, Vandervelde L, Batcho CS, et al. Can manual ability be measured with a generic ABILHAND scale? A cross-sectional study conducted on six diagnostic groups. BMJ Open 2012;2. doi:10.1136/ bmjopen-2012-001807. [Epub ahead of print: 31 Oct 2012].

43 Fabbri L, Neviani R, Festini F, et al. Transcultural validation of activities scale for kids (ASK): translation and pilot test. Acta Biomed 2016;87 Suppl 2:70-9.

44 Costi S, Mecugni D, Beccani L, et al. Construct validity of the activities scale for kids performance in children with cerebral palsy: brief report. Dev Neurorehabil 2020;23:474-7.

45 Costi S, Filippi MC, Braglia L, et al. Reliability and construct validity of the activities scale for kids in Italian children with cerebral palsy. Disabil Rehabil 2021;20:1-7.

46 Caggiano V, Fogassi L, Rizzolatti G, et al. View-based encoding of actions in mirror neurons of area $\mathrm{F} 5$ in macaque premotor cortex. Curr Biol 2011;21:144-8.

47 Ge S, Liu H, Lin P, et al. Neural basis of action observation and understanding from first- and Third-Person perspectives: an fMR study. Front Behav Neurosci 2018;12:283.

48 Cohen J. Statistical power analysis for the behavioral sciences. 2nd ed. Hillsdale, N.J: L. Erlbaum Associates, 1988.

49 Novak I, Mclntyre S, Morgan C, et al. A systematic review of interventions for children with cerebral palsy: state of the evidence. Dev Med Child Neurol 2013;55:885-910. 